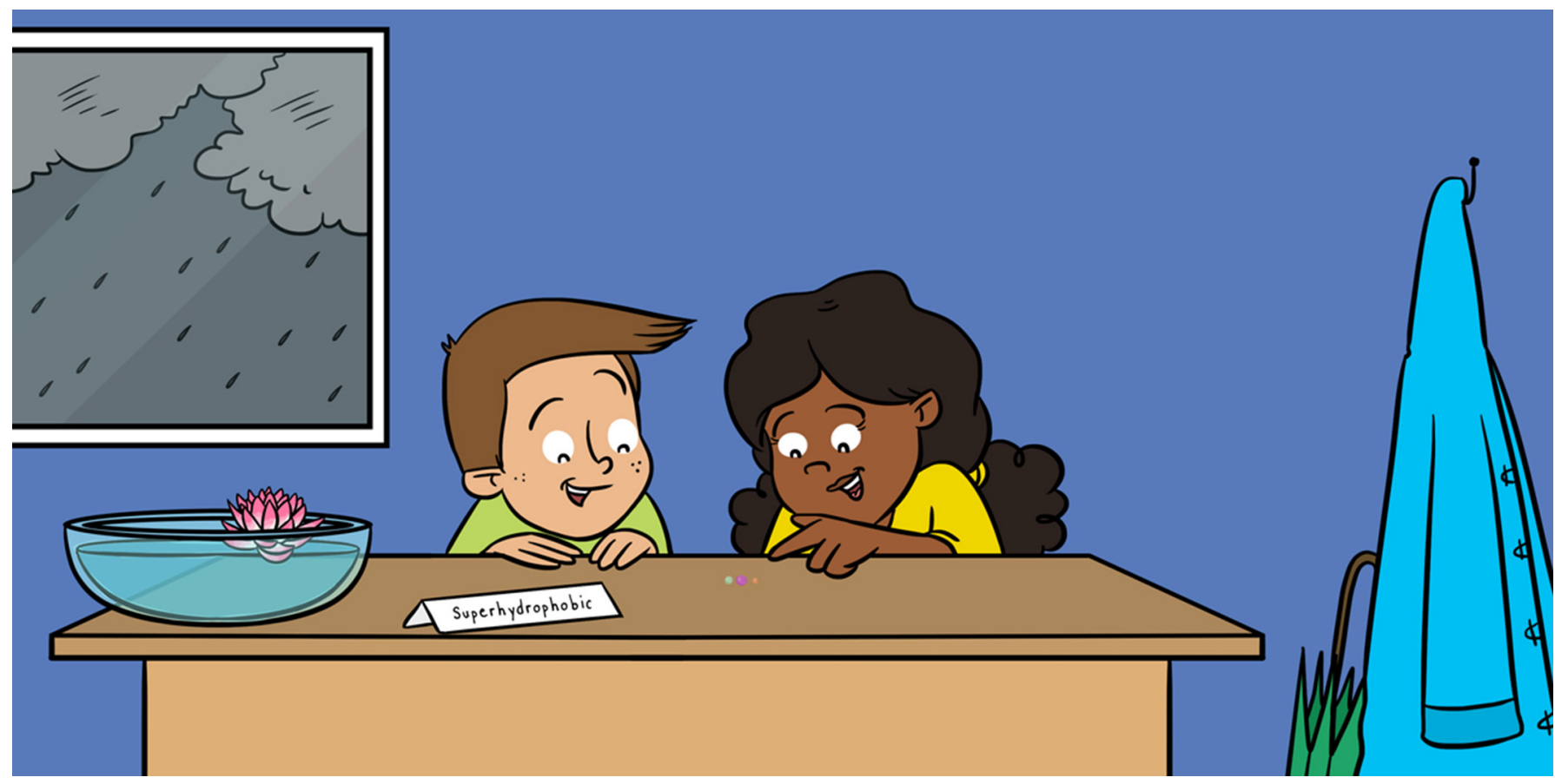

\title{
NON-WETTING MATERIALS
}

\section{Valeri Frumkin ${ }^{1 *}$ and Moran Bercovici ${ }^{2}$}

${ }^{1}$ Department of Mathematics, Massachusetts Institute of Technology, Cambridge, MA, United States

${ }^{2}$ Faculty of Mechanical Engineering, Technion-Israel Institute of Technology, Haifa, Israel

YOUNG REVIEWERS:

ORT EBIN, SCIENCE

AND

pringer TECHNOLOGY RESERVE CLASS AGES: $14-15$

\section{POLAR}

Has two poles, for example one positively charged pole and another negatively charged.
A drop of rain falling on a surface, such as the car's window or the palm of your hand, tends to spread over the surface and even hang onto it. But there are materials, such as the leaf of the lotus flower, from which a drop of water will roll down to the ground as an almost perfect sphere, while cleaning the material of dust particles and other pollutions. In this article, we will investigate what influences the tendency of different materials to be wetted to different extents, and how can we design and produce such materials in the lab.

\section{WATER IS A SPECIAL SUBSTANCE}

All substances are made up from atoms, which are the smallest unit of any element. Atoms connect to build more complex materials, called molecules. Water molecules are made from one oxygen atom and two hydrogen atoms, and the water molecule has a special structure that makes its electrical charge distribute unevenly, so that one side of the molecule is positively charged, and the other is negatively charged (Figure 1). Molecules with uneven charge distribution are called polar molecules (since they have a positive electrical side, or pole, and 
Figure 1

The structure of a water molecule. The water molecule is polar, because the side with the two hydrogen atoms has a positive charge, while the oxygen atom has a negative charge.

\section{COHESION}

The tendency of similar or identical particles or surfaces to cling to one another.

\section{ADHESION}

The tendency of dissimilar particles or surfaces to cling to one another.

\section{CONTACT ANGLE}

The angle formed at the contact line between a droplet and a solid surface, surrounded by a fluid (typically air).

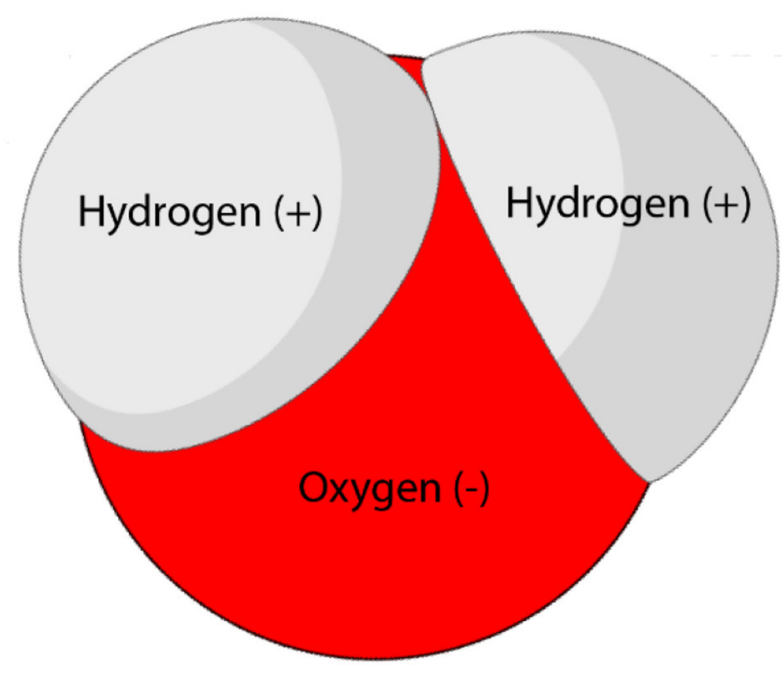

Figure 1

a negative electrical pole), and polar molecules tend to attract one another strongly. Since water is a very polar material, water molecules are strongly attracted to one another, but they are also attracted to other polar materials, and are not attracted to materials that are not polar. The attraction forces between the water molecules are called cohesion forces, and the attraction forces between water and other substances are called adhesion forces.

Let us perform an experiment: add a few drops of oil into a glass of water and look at both liquids. After a few seconds, you will see that instead of mixing with the water, the oil will form small spheres within the water, and if you wait long enough, all the oil will combine to form one continuous layer floating on top of the water. Why does this happen? Well, since oil is a non-polar material and water is a very polar material, water molecules will not be attracted to the oil molecules, but will be strongly attracted to each other, and so they will push away the oil molecules until there is a complete separation between the oil and the water. Also, since oil is less dense than water, the oil will float on top of the water.

What do you think will happen if water is put onto a piece of glass, which is another polar substance? You can probably deduce that the water molecules will be attracted to the glass molecules, and so water will spread over the glass. In contrast, for non-polar materials, such as plastic surfaces, water molecules will be attracted to each other much more strongly than they are to the plastic molecules, so a drop of water placed on a plastic surface will form the shape of half a sphere and will not spread well. To measure the amount of wetting of a certain surface, scientists measure the contact angle created between the surface and the drop that is placed on it [1] (Figure 2). If the adhesion forces are much stronger than the cohesion forces, we will get an angle smaller than $90^{\circ}$, and then we will say that the surface's material 
Figure 2

Three drops showing three contact angles. (1) The contact angle between the drop and the surface is smaller than $90^{\circ}$, which means the surface is hydrophilic. (2) The contact angle between the drop and the surface equals $90^{\circ}$. (3) The contact angle between the drop and the surface is larger than $90^{\circ}$, which means the surface is hydrophobic.

\section{HYDROPHILIC}

Water loving.

\section{HYDROPHOBIC}

Water fearing

\section{SURFACE TENSION}

The tendency of liquid surfaces to act like an elastic sheet.

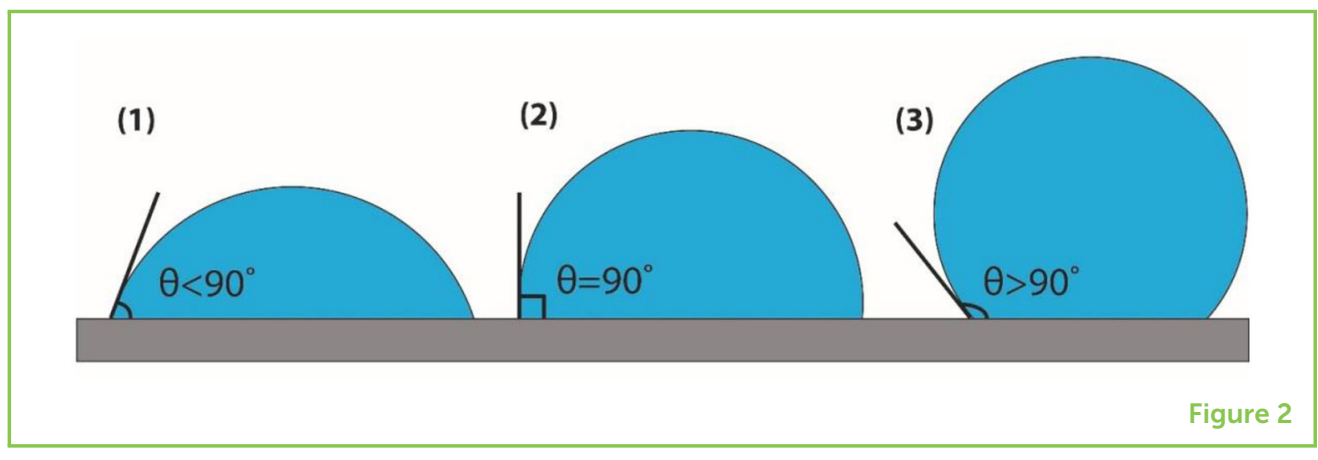

is hydrophilic (hydro = water, philic = loving). If, on the other hand, the cohesion forces are stronger than the adhesion forces, we will get a contact angle larger than $90^{\circ}$, and then we will say that the material is hydrophobic (phobic = fearing).

\section{WHY ARE DROPS SPHERICAL?}

Let us imagine a single water molecule floating in a big drop of water, surrounded in all direction by many other water molecules. Though the molecule will feel an attraction from all its friends, the net force on the molecule will be zero because it is being pulled in all directions, similar to when we pull a string with an equal force from both ends. Now, let us imagine a water molecule at the edge of the drop-above it there is air and beneath it there is water. Such a molecule will feel an attraction force toward the water beneath it, and will not feel an attraction toward the air. Imagine a line of molecules pulling on each other, leading from the edge of the drop, through its center, to the other edge. Since all the molecules are pulling each other, the line of molecules will behave like a stretched rubber band trying to contract. Since we can think of such "rubber bands" acting in all directions, all the water molecules present on the outer edge of the drop will feel a strong force pulling them toward the middle, and this force creates the surface tension of the water. This is why small water drops form the shape of a sphere-all the molecules on the edge of the drop are pulled toward the inside, creating a uniform pressure from all directions, shaping the drop into a sphere (Figure 3). This phenomenon is not unique to water. Every liquid has surface tension, but the surface tension of most liquids is usually much weaker than that of water.

The strong surface tension of water makes the surface behave like a flexible layer of rubber and even allows certain insects to walk on top of water without sinking. Moreover, if we try to distort a small water drop, the surface tension of the water will resist it and try to return the drop to its spherical shape. So, why does not water in a jug on the kitchen table have a spherical shape? It is important to remember that another force is acting on the water to flatten it, and that is the earth's gravitational force. For very small drops (like rain drops), the influence of gravity is fairly small, and that is why the drops stay spherical. However, for 
Figure 3

Three drops of water placed on a super-hydrophobic (water-repelling) surface. You can see that the biggest drop is a little squashed by gravity, while the smallest molecule keeps it spherical shape due to surface tension. Image: Ran Eshel.

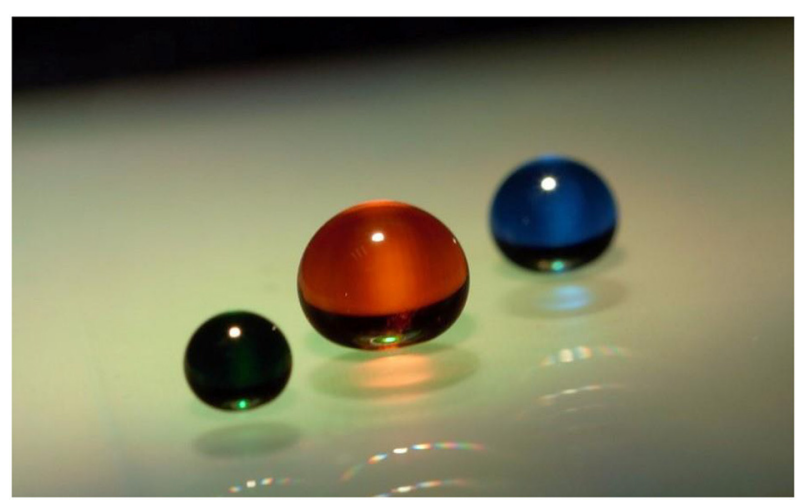

Figure 3

bodies of water bigger than a few millimeters, in which the number of molecules in the middle of the drop is significantly larger than the number of molecules on the edge, the gravitational force becomes very important, and water loses its spherical shape.

\section{HOW DO SUPER-HYDROPHOBIC SURFACES WORK?}

We mentioned that the amount of wetting of a surface can be determined by measuring the contact angle between the water and the surface. This angle is determined by the ratio between the cohesion and adhesion forces. In reality though, this is true only for perfectly smooth surfaces, while most real surfaces are rough.

In 1936, a researcher named Robert Wenzel showed that the roughness of a surface strengthens the natural wetting tendency of the material [2]. This means that if the contact angle between the water drop and the surface is slightly smaller than $90^{\circ}$ (hydrophilic surface), then the same type of material with additional roughness will have an even smaller contact angle, meaning it will be even more hydrophilic. In a similar way, if the contact angle between the water drop and the surface material is slightly larger than $90^{\circ}$ (hydrophobic surface), the roughening of the surface will create a much larger contact angle, meaning the material will be even more hydrophobic. The reason for this phenomenon is that a rough surface has more surface area, so the liquid needs to "work harder" to wet the surface it does not like, and less hard to wet the surface it does like.

In 1944, two researchers named Cassie and Baxter looked at an interesting problem of a water drop placed on a porous surface (like a sponge) [3]. They saw that if the porous material is hydrophobic and the pores are very small, then the water cannot penetrate into the pores, and the drop stays on pockets of air trapped inside the pores. It turns out that these drops easily form very large contact angles, so if we prepare a porous surface from a hydrophobic material, we will get a super-hydrophobic material! 
Figure 4

An image of a super-hydrophobic surface taken using a scanning electron microscope. This surface was produced in the lab of Prof. Bercovici at the Technion, Israel. The image shows 10 micron-wide channels (about 1/10th the diameter of human hair), which are used for trapping pockets of air. You can see small particles spread on top of the channels. These particles are about $100 \mathrm{~nm}$ long (about $1 / 1,000$ th the diameter of human hair) and are made from a special plastic materials. Their job is to prevent water from entering into the channels.

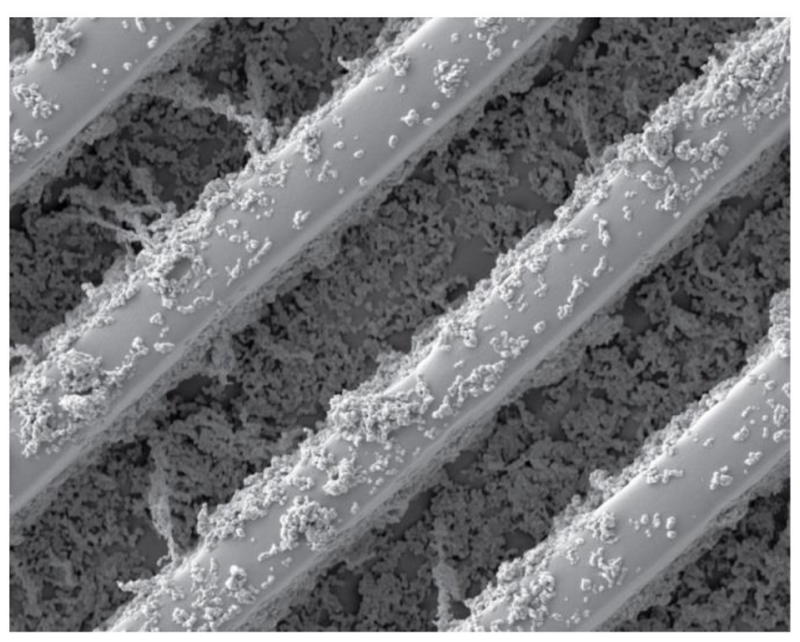

Figure 4

Super-hydrophobic materials have existed in nature for millions of years, and the most famous is the leaf of the lotus flower. If we look at this leaf through a microscope, we can see that it is covered with tiny bumps, each about one tenth the diameter of a human hair. These bumps function as pores that can trap air between them and the water. Additionally, the bumps themselves are covered in even smaller waxy hairs (each about one thousandth the diameter of a human hair), which help prevent the water from penetrating into the trapped air pockets. For a long time, we did not know how to produce materials with such tiny structures, and it was only in the late 1990's that scientists learned how to produce real super-hydrophobic materials in labs.

\section{THE FUTURE OF SUPER-HYDROPHOBIC MATERIALS}

It turns out that super-hydrophobic materials have many interesting potential applications. For example, we can use a super-hydrophobic spray to cover the windshields of vehicles to eliminate the need for windshield wipers, since drops of water will just roll off the coated windshields. The spray covers the windshields with transparent, hydrophobic microscopic particles, which give the windshield roughness and make it super-hydrophobic. Another important feature of super-hydrophobic materials is that some of them allow water to slide across them very easily, and this reduces the friction between the water and the coated object's surface. Ships and submarines with a super-hydrophobic cover will be able to move through water much more easily and will use only a fraction of the energy required today for their propulsion. The problem is that air pockets trapped between the super-hydrophobic surface and the water can collapse from factors, such as high pressure, heat, or strong water flow. Therefore, one of the major challenges in the field is producing surfaces that resist these factors and preserve their super-hydrophobic characteristics for a long time. 
In our research group, we study how we can use super-hydrophobic materials for the movement of liquids through microscopic channels. This is important for developing tiny devices that can be used to provide quick and inexpensive medical tests for everyone, including tests for things like viral or bacterial infections, early signs of a heart attack, or the effectiveness of anti-cancer drugs. A scanning electron microscope image of a super-hydrophobic surface produced in our lab, is shown in Figure 4.

The number of potential uses for super-hydrophobic materials is large and keeps growing. The market for these materials has helped this field of research to flourish, and many new discoveries are being made. It is easy to predict that we will see a greater and greater use of super-hydrophobic materials in novel technologies that will likely be a part of all of our lives in the future.

\section{REFERENCES}

1. Young, T. 1805. An essay on the cohesion of fluids. Philos. Trans. R. Soc. Lond. 95:65-87.

2. Wenzel, R. 1936. Resistance of solid surfaces to wetting by water. Ind. Eng. Chem. 28:988-94.

3. Cassie, A. B., and Baxter, S. 1944. Wettability of porous surfaces. Trans. Faraday Soc. $40: 546-51$.

SUBMITTED: 27 October 2020; ACCEPTED: 27 November 2020; PUBLISHED ONLINE: 11 December 2020.

EDITED BY: Idan Segev, Hebrew University of Jerusalem, Israel

CITATION: Frumkin V and Bercovici M (2020) Non-wetting Materials. Front. Young Minds 8:621808. doi: 10.3389/frym.2020.621808

CONFLICT OF INTEREST: The authors declare that the research was conducted in the absence of any commercial or financial relationships that could be construed as a potential conflict of interest.

COPYRIGHT (c) 2020 Frumkin and Bercovici. This is an open-access article distributed under the terms of the Creative Commons Attribution License (CC BY). The use, distribution or reproduction in other forums is permitted, provided the original author(s) and the copyright owner(s) are credited and that the original publication in this journal is cited, in accordance with accepted academic practice. No use, distribution or reproduction is permitted which does not comply with these terms. 


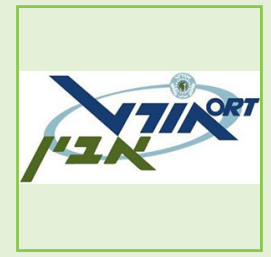

\section{YOUNG REVIEWERS}

\section{ORT EBIN, SCIENCE AND TECHNOLOGY RESERVE CLASS, AGES: 14-15}

We are a Science and Technological reserve class at the ORT Ebin school in Ramat Gan. ORT Ebin is a high school of sciences and arts. The school promotes leading technological subjects: biotechnology, robotics and computer science-cyber.

\section{AUTHORS}

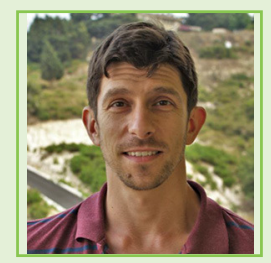

\section{VALERI FRUMKIN}

I am a post-doctoral fellow at the Mathematics department at the Massachusetts Institute of Technology, formerly from Technion-Israel Institute of Technology. I am very interested in interfacial phenomena, which is all the strange and surprising stuff that happens at the boundaries between different fluids. I also love communicating science to the general audience and sharing all the beautiful things we get to see and discover. *valerafr@gmail.com

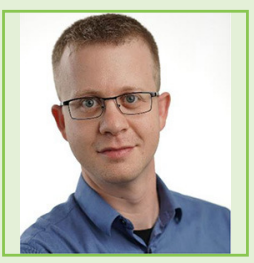

\section{MORAN BERCOVICI}

I am an Associate Professor of Mechanical and Biomedical Engineering at Technion-Israel Institute of Technology. In my lab we study how liquids behave at the microscale, when they are affected by thermal and electric fields, chemical reactions, and biological processes. We are interested in understanding basic physical mechanisms and in leveraging them to create new tools and technologies across different disciplines. 